\title{
Binary Stars and Globular Cluster Dynamics
}

\author{
John M. Fregeau ${ }^{1}$ \\ Northwestern University, Department of Physics and Astronomy, Evanston, IL 60208, USA. \\ email: fregeau@northwestern.edu \\ ${ }^{1}$ Chandra Fellow
}

\begin{abstract}
In this brief proceedings article I summarize the review talk I gave at the IAU 246 meeting in Capri, Italy, glossing over the well-known results from the literature, but paying particular attention to new, previously unpublished material. This new material includes a careful comparison of the apparently contradictory results of two independent methods used to simulate the evolution of binary populations in dense stellar systems (the direct $N$-body method of Hurley, Aarseth, \& Shara (2007) and the approximate Monte Carlo method of Ivanova et al. (2005)), that shows that the two methods may not actually yield contradictory results, and suggests future work to more directly compare the two methods.
\end{abstract}

Keywords. globular clusters: general, open clusters and associations: general, binaries: general, methods: n-body simulations, stellar dynamics

\section{Preamble}

As this conference is an occasion to celebrate Douglas Heggie's 60th birthday, I did the following to honor him. For each slide in my presentation for which Douglas had some impact - by directly working on a topic, by influencing the way people think about a topic, or by influencing my own personal thinking on a topic - I colored the slide background white. Interestingly, every single slide in my presentation had a white background.

\section{Introduction}

Globular clusters are observed to contain significant numbers of binary star systems - so many, in fact, that they must have born with binaries (Hut et al. 1992). Their presence in clusters is important for two complementary reasons. Through super-elastic dynamical scattering interactions, they act as an energy source which may postpone core collapse, and may be the dominant factor in setting the core radii of observed Galactic globulars. Similarly, the dense stellar environment and increased dynamical interaction rate in cluster cores is responsible for the high specific frequency of stellar "exotica" found in clusters, including low-mass X-ray binaries (LMXBs), cataclysmic variables $(\mathrm{CVs})$, blue straggler stars (BSSs), and recycled millisecond pulsars (MSPs).

\section{Evolution of Clusters}

\subsection{The Negative Heat Capacity of Self-Gravitating Systems}

Imagine finding yourself piloting a spaceship in orbit about a planet. Your ship is equipped with rocket thrusters that can either fire in your direction of motion or opposite it. If you want to slow down, which way do you fire your thrusters?

The answer is that you fire your thrusters behind you - in your direction of motion. This causes work to be done on your spaceship, which increases your energy, expands your orbit about the planet, and slows you down. It's counterintuitive at first, since it's 
like depressing the accelerator pedal to slow down, but this behavior is typical of selfgravitating systems, and is a manifestation of their negative heat capacity - if you add energy to a system it cools down, if you take energy away from a system is heats up. A real-life example of this is air drag on an orbiting satellite, which will actually cause it to speed up.

A basic appreciation of the negative heat capacity of self-gravitating systems goes a long way in helping to understand the physics of the binary burning phase in clusters (the phase analogous to the main sequence in stars, in which clusters "burn" binaries instead of hydrogen to support their cores against collapse). Imagine a binary star system in a cluster encountering a single star, where the relative speed between the binary and the single star is smaller than the orbital speed in the binary system. When the three stars get close enough to interact strongly, the quickly moving binary members will tend to transfer some energy to the more slowly moving incoming single star (energy transfer from hot to cold). The result is that when the interaction is over one of the three stars (and it doesn't have to be the original single star) will leave with a higher relative velocity than the incoming single star initially had. Since the binary system gave up some energy to the single in the interaction it will become more tightly bound and thus have a larger orbital speed (energy was taken from it and it got hotter). The binary we have constructed in this thought experiment is a "hard" binary (since the orbital speed in the binary is larger than the encounter speed), which clearly becomes harder as a result of dynamical interactions (Heggie 1975)p. In general, a population of hard primordial binaries will act as an energy source that supports a cluster's core against collapse through dynamical scattering interactions (please see Heggie \& Hut (2003) for a more detailed discussion).

It's easy to make an order of magnitude estimate of the importance of binaries in a cluster (the following discussion closely follows that in Heggie \& Hut (2003)). Imagine a cluster with $N$ objects, $f_{b} N$ of which are hard binaries. Denote the total cluster mechanical energy as $E_{\text {ext }}$, and the total binary binding energy as $E_{\text {int }}$. The binding energy of a binary with hardness $x$ is then

$$
E_{b} \equiv x k T \approx x E_{\mathrm{ext}} / N
$$

where $k T$ represents thermal energy of motion. The total internal energy is then

$$
E_{\mathrm{int}} \approx f_{b} x E_{\mathrm{ext}} .
$$

Since a binary releases energy of order $E_{b}$ through interactions, binaries are important when $f_{b} x \gtrsim 1$. For example, for binaries of hardness $x=10$ (a reasonable value), a binary fraction of merely $10 \%$ can be enough to unbind a cluster completely. It would also appear that just one sufficiently hard binary could be dynamically very important. However, it should be noted that one key element has been left out of the discussion: interaction timescales. A very hard binary composed of stars that are roughly the average stellar mass in the cluster would have such a small semi-major axis as to make its interaction time so long that it is essentially dynamically irrelevant.

A more detailed analysis of energy generation due to binary burning can give a rough estimate of the equilibrium core radius in the binary burning phase, and can be compared with observations (the following discussion closely follows that in Goodman \& Hut (1989)). In equilibrium the energy generated in the core via binary burning should equal the energy transported across the half-mass radius via two-body relaxation. The binary burning energy generation rate is

$$
\dot{E}_{\mathrm{bin}} \approx n_{c}\left(n_{c} \sigma_{\mathrm{bin}} v_{c}\right)\left(\frac{4 \pi r_{c}^{3}}{3}\right)\left(\frac{G m^{2}}{2 a}\right) \sim r_{c}^{3} n_{c}^{2} \frac{G^{2} m^{3}}{v_{c}} g\left(f_{b}, A_{\mathrm{bb}}, A_{\mathrm{bs}}\right),
$$


where $n_{c}$ is the core number density, the first term in parentheses is the $n-\sigma-v$ estimate for the interaction rate of a binary, the second term in parentheses is the core volume, and the third term is the binding energy of a typical binary. The function $g\left(f_{b}, A_{\mathrm{bb}}, A_{\mathrm{bs}}\right)$ is a dimensionless function of the binary fraction, the relative strengths of binary-binary and binary-single energy generation, and is of order unity. The two-body relaxation energy transport rate is

$$
\dot{E}_{\mathrm{rel}}=\frac{|E|}{\alpha t_{\mathrm{rh}}} \approx \frac{1}{5 \alpha} \frac{G M^{2}}{t_{\mathrm{rh}} r_{h}},
$$

where $\alpha$ is a constant, $t_{\mathrm{rh}}$ is the relaxation time at the half-mass radius, and $r_{h}$ is the half-mass radius. Equating the two expressions yields

$$
\frac{r_{c}}{r_{h}} \approx \frac{0.05}{\log _{10}(\gamma N)} g\left(f_{b}, A_{\mathrm{bb}}, A_{\mathrm{bs}}\right),
$$

where the standard expression has been substituted in for the relaxation time, with $\log _{10}(\gamma N)$ the Coulomb logarithm. For $N=10^{6}$ this expression yields $r_{c} / r_{h} \sim 0.02$ which is in rough agreement only with the $\sim 20 \%$ of Galactic globulars that are observationally classified as core collapsed.

\subsection{Globular Cluster Core Radii}

Recently two independent and very different numerical methods for simulating the evolution of star clusters have been used to study the core radii of clusters in the binary burning phase. One is the direct $N$-body method, which utilizes very few approximations and thus treats the evolution of clusters on a dynamical (orbital) timescale. The other is the Monte Carlo method, which uses a number of assumptions in order to treat the evolution on a relaxation timescale. To accurately treat them, binary interactions are handled via direct few-body integration. Remarkably, the two methods agree quite well in the value of $r_{c} / r_{h}$ predicted during the binary burning phase (Heggie, Trenti, \& Hut 2006; Fregeau \& Rasio 2007). Unfortunately, the value predicted by the simulations is at least an order of magnitude smaller than what's observed for the $\sim 80 \%$ of clusters that are observed to be non-core collapsed. Since the longest phase of evolution for a cluster is the binary burning phase, it is expected that most clusters currently observed should be in this phase. The current state of the field thus represents a major discrepancy between theory and observations.

Several resolutions to the problem have been proposed. Hurley (2007), among others, has noted that there are in fact three different definitions of the core radius in popular use, with the observational definition possibly being larger than the standard dynamical definition used in some numerical codes by a factor of $\sim 4$. Another suggestion is that there are central intermediate-mass black holes (IMBHs) in most Galactic globular clusters, which act as an energy source that would increase core radii to roughly the value observed (Trenti 2006; Miocchi 2007; Trenti et al. 2007). It could also be that the "true" initial conditions for clusters are of much higher or lower stellar density than what has traditionally been assumed in simulations (Fregeau \& Rasio 2007). Or it could be that stellar mass loss from enhanced stellar evolution of physical collision products could power the cores sufficiently (Chatterjee et al., this volume). For young clusters, mass segregation of compact remnants (Merritt et al. 2004), or the evaporation of the sub-population of stellar-mass black holes (Mackey et al. 2007) could possibly explain the discrepancy. 


\subsection{Globular Cluster Binary Fractions}

Observations show that globular clusters currently have core binary fractions ranging from a few \% (NGC 6397, 47 Tuc, M4), to 30\% (Pal 13, E3, NGC 6752, NGC 288). The observational techniques used to determine the core binary fractions are varied, but include observation of a secondary main sequence, radial velocity studies, and searches for eclipsing binaries. All methods involve extrapolation of somewhat uncertain functions (e.g., the binary mass ratio distribution in the first method), although the first method is considered to be the most complete. When combined with observations of other cluster properties, measured core binary fractions enable detailed testing of cluster evolution models.

Two key processes govern the evolution of the binary fraction in clusters: binary stellar evolution, and stellar dynamical interactions. Two codes currently combine both processes to varying degrees of realism. The work of Hurley, Aarseth, \& Shara (2007) uses full $N$-body calculations with binary stellar evolution. That of Ivanova et al. (2005) uses binary stellar evolution with a simplified dynamical model that assumes a two-zone cluster (core and halo) with constant core density, but performs direct few-body integration of binary scattering interactions. In perhaps oversimplified terms, Ivanova et al. (2005) find generally that the core binary fraction in clusters decreases with time, requiring clusters to have been born with large binary fractions to explain currently observed binary fractions. In similarly oversimplified terms, Hurley, Aarseth, \& Shara (2007) generally find that the core binary fraction tends to increase with time. This is certainly a simplified comparison of the two apparently contradictory results, since there are several differences in initial conditions and assumptions used by the two different methods that need to be taken into account. The dynamics and evolution of binary populations in clusters - especially when coupled with a realistic treatment of binary stellar evolution - is a complex topic, however, and the important differences between the two methods and the clusters they model sometimes get lost in the discussion.

One of the most important parameters governing the evolution of binary fractions in clusters is the stellar density in the core. A larger density leads to a higher dynamical interaction rate, and it is these interactions that can affect stellar evolutionary processes by modifying orbital parameters of binaries, destroying binaries, exchanging members of binaries, and creating binaries via tidal capture. Fig. 1 shows the evolution of cluster evolution models in core number density and core binary fraction for the $N$-body simulations of Hurley, Aarseth, \& Shara (2007) (solid arrows) and the Monte Carlo simulations of Ivanova et al. (2005) (dashed arrows). Note that the binary fraction plotted here is the hard binary fraction. The models of Hurley, Aarseth, \& Shara (2007) use exclusively hard binaries, while those of Ivanova et al. (2005) start with a wide distribution of binaries that extends well into the soft regime. The globular clusters for which the core number density and binary fraction are known are plotted as open circles. The single triangle point represents a typical open cluster. In comparing the two models, a clear sign of fundamental disagreement would be if an $N$-body arrow and a Monte Carlo arrow begin in the same region of phase space but point in different directions. Only the two arrows at $n_{c}=10^{3} \mathrm{pc}^{-3}$ show this disagreement, pointing in opposite directions. However, the Monte Carlo method is less accurate at this low stellar density, since the assumption of constant core density breaks down here. Similarly, the $N$-body arrow appears to be horizontal since the initial and final densities are not known - only the average density is known for this model. In other words, the only two contradictory arrows in this diagram may not faithfully represent their respective methods. All other arrows are in very different areas of parameter space, and so unfortunately do not offer direct comparisons 


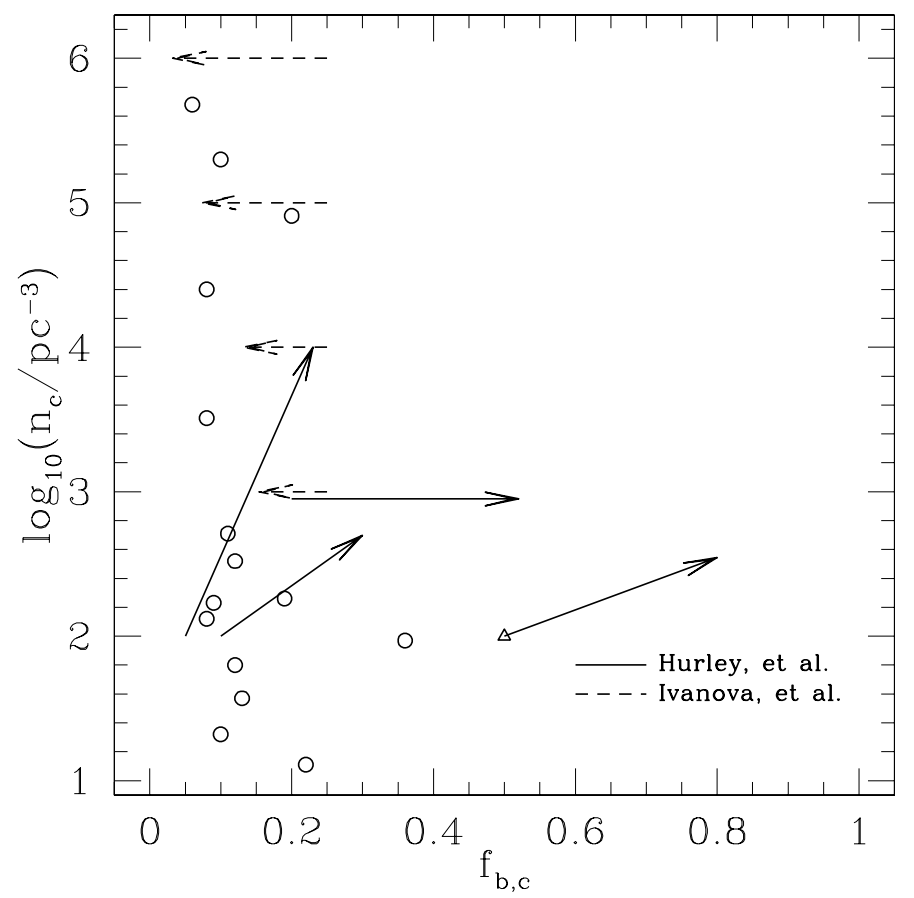

Figure 1. The evolution of cluster evolution models in core number density and core binary fraction for the $N$-body simulations of Hurley, Aarseth, \& Shara (2007) (solid arrows) and the Monte Carlo simulations of Ivanova et al. (2005) (dashed arrows). Note that the binary fraction plotted here is the hard binary fraction. The models of Hurley, Aarseth, \& Shara (2007) use exclusively hard binaries, while those of Ivanova et al. (2005) start with a wide distribution of binaries that extends well into the soft regime. The globular clusters for which the core number density and binary fraction are known are plotted as open circles. The single triangle point represents a typical open cluster.

between the two methods. $N$-body simulations are generally relegated to clusters with rather low initial densities $\left(\lesssim 10^{3} \mathrm{pc}^{-3}\right)$, while the Monte Carlo simulations are most accurate for rather higher densities $\left(\gtrsim 10^{3} \mathrm{pc}^{-3}\right)$. Thus it could very well be that the results of both methods represent the same underlying evolution. In order to fully compare the methods either many more Monte Carlo simulations should be performed with lower initial densities (although the assumption of constant core density breaks down as the density becomes lower), or $N$-body simulations should be performed for larger initial density and binary fraction (although this is currently quite computationally expensive).

A viable alternative to properly compare the two methods is to perform an $N$-body simulation at large initial core density for just a very short time to get a sense of the direction of evolution in phase space. It should be noted that the arrows in Fig. 1 are straight-line approximations to the true evolution. Thus it should first be tested whether the overall evolution of an $N$-body model in this parameter space has any relation to the initial, differential evolution.

\section{Evolution of the Binary Population}

Due to strong binary scattering interactions, globular clusters are home to large numbers of "exotic" stellar objects, including LMXBs, CVs, MSPs, and BSSs. The 
interactions can create and destroy classes of binaries directly through exchange and ionization, and indirectly by modifying orbital properties and thus affecting binary stellar evolution processes.

\subsection{The Interaction Frequency for X-Ray Sources}

It was realized over 30 years ago that globular clusters are overabundant per unit mass in X-ray binaries by orders of magnitude relative to the disk population (Clark 1975; Katz 1975). If X-ray binaries are formed in clusters mainly via binary scattering interactions, there should be a correlation between the interaction rate and the number of such binaries in each cluster (Verbunt \& Hut 1987). Since the interaction rate is so heavily used in the literature, it is worthwhile to review its derivation.

Imagine a large volume of uniform density $n_{1}$ of an object labeled type 1 , and similarly for type 2. The interaction rate for one member of species 2 with species 1 is

$$
\frac{d N_{2}}{d t}=n_{1} \sigma_{12} v_{12}
$$

where $\sigma_{12}$ is the cross section for interaction of an object of species 1 with an object of species 2 , and $v_{12}$ is the relative speed between the two species. The total interaction rate per unit volume is then

$$
\frac{d N_{\mathrm{int}}}{d t d V}=n_{1} n_{2} \sigma_{12} v_{12}
$$

which is nicely symmetric under transformation between index 1 and 2. The total interaction rate for a cluster can be approximated by multiplying by the core volume, to give

$$
\Gamma \equiv \frac{d N_{\mathrm{int}}}{d t} \approx n_{1} n_{2} \sigma_{12} v_{12} \frac{4 \pi r_{c}^{3}}{3} \propto \rho^{2} r_{c}^{3} / v_{\sigma},
$$

where $r_{c}$ is the core radius, $\rho$ is the core mass density, and $v_{\sigma}$ is the core velocity dispersion. The last proportionality involves, among other things, substituting in the mass density and using the gravitational focusing dominated interaction cross section. $\Gamma$, in various incarnations, has been used for many years in analyses comparing with the observed numbers of X-ray sources in clusters. Recently, Pooley et al. (2003) have shown that the number of observed X-ray sources above $4 \times 10^{30} \mathrm{erg} / \mathrm{s}$ in the $0.5-6 \mathrm{keV}$ range displays a stronger correlation with cluster $\Gamma$ than with any other cluster parameter.

The fact that the number of X-ray sources in clusters so strongly correlates with $\Gamma$ is surprising given the number of approximations that go into deriving it. For example, the two interacting populations in eq. (4.3) are compact objects (neutron stars and white dwarfs) and stellar binaries, whose densities may differ dramatically. The use of $\rho$ in place of $n_{i}$ assumes a proportionality between the stellar density and the compact object density that is constant among all clusters. Furthermore, there is a factor of $f_{b}$, the binary fraction, that has been dropped, thereby implicitly assuming that it is constant among all clusters. There is also a factor of the binary semi-major axis that has been dropped from the variation, which may vary since the hard-soft binary boundary will vary among clusters. In addition, the recent dynamical history of the cluster may play an important role.

Improvements to the standard $\Gamma$ analysis have recently been made. One of the difficulties present in the earlier analyses is that $\Gamma$ strongly correlates with total cluster mass. This is unfortunate, since it makes it more difficult to distinguish dynamically-formed sources (whose number should correlate with $\Gamma$ ), from primordial sources (whose number should correlate with total cluster mass). An analysis of the CV populations in clusters 
using a normalized interaction rate, $\gamma \equiv \Gamma / M_{\text {clus }}$, has shown that like LMXBs, CVs are predominantly formed via dynamical encounters (Pooley \& Hut 2006).

\section{Summary}

In this proceedings article I have very briefly discussed the connection between binary stars and globular cluster dynamics, moving from basic physics to current research in the span of a few paragraphs. A thorough, easily readable, and fairly recent discussion of the material can be found in Heggie \& Hut (2003).

The primary new material presented here is a comparison in phase space of the seemingly contradictory binary population evolution simulations of Ivanova et al. (2005) and Hurley, Aarseth, \& Shara (2007), showing that they may in fact both represent the same underlying physics. In other words, new simulations must be performed to better compare the two very different methods.

\section{Acknowledgements}

For data, stimulating discussions, and general camaraderie, the author thanks Craig Heinke, Jarrod Hurley, Natasha Ivanova, Frederic Rasio, M. Atakan Gürkan, and Marc Freitag. JMF acknowledges support from Chandra theory grant TM6-7007X, as well as Chandra Postdoctoral Fellowship Award PF7-80047.

\section{References}

Clark, G. W. 1975, ApJL, 199, L143

Fregeau, J. M. \& Rasio, F. A. 2007, ApJ, 658, 1047

Goodman, J. \& Hut, P. 1989, Nature, 339, 40

Heggie, D. C. $1975, M N R A S, 173,729$

Heggie, D. \& Hut, P. 2003, The Gravitational Million-Body Problem: A Multidisciplinary Approach to Star Cluster Dynamics, by Douglas Heggie and Piet Hut. Cambridge University Press, 2003, 372 pp.

Heggie, D. C., Trenti, M., \& Hut, P. 2006, MNRAS, 368, 677

Hurley, J. R. 2007, MNRAS, 379, 93

Hurley, J. R., Aarseth, S. J., \& Shara, M. M. 2007, ApJ, 665, 707

Hut, P., et al. 1992, PASP, 104, 981

Ivanova, N., Belczynski, K., Fregeau, J. M., \& Rasio, F. A. 2005, MNRAS, 358, 572

Katz, J. I. 1975, Nature, 253, 698

Mackey, A. D., Wilkinson, M. I., Davies, M. B., \& Gilmore, G. F. 2007, MNRAS, 379, L40

Merritt, D., Piatek, S., Portegies Zwart, S., \& Hemsendorf, M. 2004, ApJL, 608, L25

Miocchi, P. 2007, MNRAS, 783

Pooley, D., et al. 2003, ApJL, 591, L131

Pooley, D., \& Hut, P. 2006, ApJL, 646, L143

Trenti, M. 2006, ArXiv Astrophysics e-prints, arXiv:astro-ph/0612040

Trenti, M., Ardi, E., Mineshige, S., \& Hut, P. 2007, MNRAS, 374, 857

Verbunt, F. \& Hut, P. 1987, The Origin and Evolution of Neutron Stars, 125, 187 